Pacific

Journal of

Mathematics

A CURVATURE FLOW UNIFYING SYMPLECTIC CURVATURE FLOW AND PLURICLOSED FLOW

SONG DAI

Volume $277 \quad$ No. 2

October 2015 


\title{
A CURVATURE FLOW UNIFYING SYMPLECTIC CURVATURE FLOW AND PLURICLOSED FLOW
}

\author{
SONG DAI
}

\begin{abstract}
Streets and Tian $(2010,2014)$ introduced pluriclosed flow and symplectic curvature flow. Here we construct a curvature flow to unify these two flows. We show the short-time existence of our flow and exhibit an obstruction to long-time existence.
\end{abstract}

\section{Introduction}

In recent years, Streets and Tian initialized the study of special geometric structures, such as generalized Kähler and symplectic structures, by using curvature flows they introduced. They include Hermitian curvature flow, pluriclosed flow, almost Hermitian curvature flow and symplectic curvature flow [Streets and Tian 2010; 2011; 2014]. Subsequently, there are several further works along this direction; see [Boling 2014; Enrietti et al. 2015; Enrietti 2013; Fernández-Culma 2013; Pook 2012; Smith 2013; Streets and Tian 2013; 2012; Vezzoni 2011]. In this paper, we introduce a curvature flow which unifies symplectic curvature flow and pluriclosed flow.

Streets and Tian [2014] introduced symplectic curvature flow, which preserves almost Kähler structure, as follows:

$$
\begin{aligned}
\frac{\partial}{\partial t} g & =-2 \operatorname{Ric}+\frac{1}{2} B^{1}-B^{2}, \\
\frac{\partial}{\partial t} J & =\triangle J+\mathcal{N}+\mathscr{R}, \\
g(0) & =g_{0}, \\
J(0) & =J_{0},
\end{aligned}
$$

where $\mathscr{R}$ is a curvature term and $B^{1}, B^{2}, \mathcal{N}$ are all quadratic terms of $D J$. We will give the precise definitions of these tensors in Section 3.

Streets and Tian [2010] introduced pluriclosed flow, which preserves pluriclosed

The author is partially supported by China Scholarship Council.

MSC2010: 53C15, 53C44, 53C56, 53D05, 53D15.

Keywords: symplectic curvature flow, pluriclosed flow. 
structure, as follows:

$$
\begin{aligned}
\frac{\partial}{\partial t} \omega & =\partial \partial^{*} \omega+\bar{\partial} \bar{\partial}^{*} \omega+\frac{1}{2} \sqrt{-1} \partial \bar{\partial} \log \operatorname{det} g, \\
\omega(0) & =\omega_{0} .
\end{aligned}
$$

Then, in [Streets and Tian 2013; 2012] they observed that, after a gauge transformation induced by the Lee form $\theta=-J d^{*} \omega$, pluriclosed flow is equivalent to the following flow:

$$
\begin{aligned}
\frac{\partial}{\partial t} g & =-2 \text { Ric }+\frac{1}{2} \mathscr{B}, \\
\frac{\partial}{\partial t} J & =\triangle J+\mathscr{R}+2, \\
g(0) & =g_{0}, \\
J(0) & =J_{0},
\end{aligned}
$$

where $\mathscr{B}$ and 2 are quadratic terms of $D J$. We will give the precise definitions of these tensors in Section 3. In this setting, they showed that twisted generalized Kähler manifolds are a natural background in which to run pluriclosed flow [Streets and Tian 2012].

Hitchin [2003] first introduced the notion of generalized complex structure, which unifies symplectic structure and complex structure. After that, Gualtieri discussed generalized complex structure in detail in his thesis [Gualtieri 2011]. In that work, Gualtieri discovered that a pair of compatible almost generalized complex structures $\left(\mathscr{F}_{1}, \mathscr{F}_{2}\right)$ is equivalent to almost bi-Hermitian data $\left(g, J_{+}, J_{-}, b\right)$, where $J_{ \pm}$are almost complex structures, compatible with $g$, and $b$ is a 2-form. If $\mathscr{f}_{1}, \mathscr{f}_{2}$ are both integrable, i.e., generalized Kähler, the integrability condition is equivalent to

$$
\begin{aligned}
N_{J_{+}} & =N_{J_{-}}=0, \\
-d_{+}^{c} \omega_{+} & =d_{-}^{c} \omega_{-}=d b .
\end{aligned}
$$

If we only require $d b$ to be a closed 3-form $H$ (which is the twisted case) Streets and Tian [2012] showed that the equivalent pluriclosed flow (2) of $\left(g, J_{+}\right)$and $\left(g, J_{-}\right)$preserves generalized Kähler structure.

A symplectic structure $\omega$ gives a generalized complex structure $\mathscr{F}_{\omega}$, and an almost Kähler structure $(\omega, J)$ gives a compatible pair of almost generalized complex structures $\left(\mathscr{F}_{\omega}, \mathscr{F}_{J}\right)$, where $\mathscr{F}_{\omega}$ is integrable while $\mathscr{F}_{J}$ is not necessarily. So one may also regard symplectic curvature flow as a curvature flow to deform a compatible pair of almost generalized complex structures $\left(\mathscr{F}_{1}, \mathscr{E}_{2}\right)$, where $\mathscr{F}_{1}$ is integrable. This leads to the question of whether or not there is a curvature flow that unifies the flows in (1) and (2). The following theorem gives a solution to this problem. 
Theorem 1.1. Let $\left(M, g_{0}, J_{0}\right)$ be an almost Hermitian manifold. Suppose $M$ is compact. Then there exists a unique family of almost Hermitian structures $(g(t), J(t)), t \in[0, \epsilon)$ on $M$ satisfying the equations

$$
\begin{aligned}
\frac{\partial}{\partial t} g & =-2 \text { Ric }+Q_{1}, \\
\frac{\partial}{\partial t} J & =\triangle J+\mathcal{N}+\mathscr{R}+Q_{2}, \\
g(0) & =g_{0}, \\
J(0) & =J_{0} .
\end{aligned}
$$

Here $\mathscr{R}$ and $\mathcal{N}$ are the same as in (1), and $Q_{1}$ and $Q_{2}$ are quadratic terms of $D J$ (see Section 3 for their precise definitions). This flow preserves the integrability of $J$. Furthermore, if the initial data is almost Kähler, this flow coincides with symplectic curvature flow, and if the initial data is pluriclosed, this flow is equivalent to pluriclosed flow. In particular, if the initial data is Kähler, this flow is Kähler-Ricci flow.

Another motivation to unify (1) and (2) is to try to understand symplectic curvature flow better. The tremendous success of [Perelman 2002] motivates us to find similar tools in symplectic curvature flow as exist in Ricci flow. To begin with, we consider whether symplectic curvature flow is a gradient flow, as is Ricci flow. It seems difficult to construct such a functional directly. But as shown in [Streets and Tian 2013], pluriclosed flow is a gradient flow, and the functional is similar to the case of Ricci flow. So maybe our flow could give some hints to discover the desired functional in symplectic curvature flow.

Turning to regularity, we derive the evolution equations, and then obtain the derivative estimates, as follows.

Theorem 1.2. Let $(M, g(t), J(t))$ be a solution of (3) for $t \in[0, T)$. Suppose $M$ is compact. If there exists a constant $K$ such that

$$
\sup _{[0, T) \times M}\left\{t|\mathrm{Rm}|, t^{1 / 2}|D J|\right\} \leq K,
$$

then for $k \geq 0$ there exists a constant $C=C(k, n, K)$ such that

$$
\sup _{[0, T) \times M}\left\{t^{(k+2) / 2}\left|D^{k} \mathrm{Rm}\right|, t^{k / 2}\left|D^{k} J\right|\right\} \leq C .
$$

Finally, we obtain an obstruction to long-time existence.

Theorem 1.3. Let $(M, g(t), J(t))$ be a solution of (3) for $t \in[0, T)$, and let $T<$ $+\infty$ be the maximal existence time. Suppose $M$ is compact. Then

$$
\sup _{[0, T) \times M}\{|\operatorname{Rm}|,|D J|\}=+\infty .
$$


We outline the proof now. Some results in this paper can be implied directly from the results in [Streets and Tian 2014]. For the convenience of readers, we give the complete proof here.

To prove Theorem 1.1, we use the DeTurck trick. But we notice that the almost complex structure $J$ does not live in a vector space. So we transform the equation on the space of almost complex structures to its tangent space at $J_{0}$. We don't assume $(g, J)$ is compatible at first, so we do some modifications to ensure the compatibility, which gives the nondegenerate symbol. Thus we obtain the short-time existence of the modified flow. Then we do some estimates to show that the modified flow gives a compatible pair $(g, J)$ and that it coincides with the initial flow. For uniqueness, it is the same as in Ricci flow. In the symplectic and pluriclosed settings, by direct calculation in Section 3 we see that this flow can be reduced to symplectic curvature flow and pluriclosed flow, respectively. So, by uniqueness, they coincide with our flow. And a similar argument also applies to the integrability of $J$.

To prove Theorem 1.2, the argument is standard. We derive the evolution equations of $D^{k} J$ and $D^{k} \mathrm{Rm}$, then we construct a function involving the terms we want to estimate. Calculating the evolution equation of this function, and then using the maximum principle, we obtain the desired result. To prove Theorem 1.3, the argument is also standard and the same as in Ricci flow.

We organize the paper as follows. In Section 2, we recall some preliminaries in almost Hermitian geometry and derive the necessary condition of a variation of almost Hermitian pairs. In Section 3 we define the tensors we will use in this paper. Then we do some calculations to show that our flow satisfies the necessary condition. And, also by calculation, we show that the additional tensors will vanish in special cases. In Section 4, we prove Theorem 1.1. In Section 5, we prove Theorem 1.2 and Theorem 1.3.

\section{Preliminaries}

We fix some conventions first.

Convention. (i) Let $g$ be a Riemannian structure. We identify elements $T \in$ $\Gamma(\operatorname{End}(T M))$ and $T \in \Gamma\left(T^{*} M \otimes T^{*} M\right)$ by

$$
g(T(X), Y)=T(X, Y) .
$$

We implicitly use this identification throughout this paper.

(ii) When we write repeated indices, we always mean to take the trace with respect to these two positions, i.e., to choose an orthonormal basis and take the sum.

(iii) We write $D J^{* 3}$ for $D J * D J * D J$, etc.

(iv) Sometimes we write $i$ instead of $e_{i}$ for short. 
(v) Sometimes we omit the time parameter $t$ if there is no ambiguity.

(vi) $D$ denotes the Levi-Civita connection, which we always use throughout the paper.

We come back to the preliminaries.

Let $M$ be a manifold, $J$ be a section of End(TM). We call $J$ an almost complex structure if $J^{2}=-1$. An almost complex structure $J$ is called integrable if $J$ is induced by holomorphic coordinates. By the theorem of Newlander and Nirenberg [1957], $J$ is integrable if and only if $N=0$, where

$$
N(X, Y)=[J X, J Y]-[X, Y]-J[J X, Y]-J[X, J Y]
$$

is called the Nijenhuis tensor.

We call $(g, J)$ an almost Hermitian structure if $g$ is a Riemannian metric, $J$ is an almost complex structure and $(g, J)$ is compatible, meaning that

$$
g(J X, J Y)=g(X, Y) .
$$

For almost Hermitian structure $(g, J)$, we define

$$
\omega(X, Y)=g(J X, Y) .
$$

Moreover, if $J$ is integrable, $(g, J)$ is called a Hermitian structure. If $d \omega=0$, then $(g, J)$ is called an almost Kähler structure. If $J$ is integrable and $d \omega=0$, then $(g, J)$ is called a Kähler structure. If $J$ is integrable and $d d^{c} \omega=0$, where

$$
d^{c} \omega(X, Y, Z):=-d \omega(J X, J Y, J Z),
$$

then $(g, J)$ is called a pluriclosed or SKT structure (strong Kähler with torsion).

Definition 2.1. Let $h \in \Gamma\left(T^{*} M \otimes T^{*} M\right)$. We define

$$
\begin{aligned}
h^{\text {sym }}(X, Y) & =\frac{1}{2}(h(X, Y)+h(Y, X)), \\
h^{\text {skew }}(X, Y) & =\frac{1}{2}(h(X, Y)-h(Y, X)) .
\end{aligned}
$$

Definition 2.2. Let $(g, J)$ be an almost Hermitian structure. Let $h \in \Gamma\left(T^{*} M \otimes T^{*} M\right)$. We define

$$
\begin{aligned}
h^{(1,1)}(X, Y) & =\frac{1}{2}(h(X, Y)+h(J X, J Y)), \\
h^{(0,2)+(2,0)}(X, Y) & =\frac{1}{2}(h(X, Y)-h(J X, J Y)) .
\end{aligned}
$$

We say that $h$ is $(1,1)$ or $(0,2)+(2,0)$ if $h^{(0,2)+(2,0)}=0$ or $h^{(1,1)}=0$, respectively.

In Lemma 2.3 and Lemma 2.6, we derive the necessary condition of a variation of almost Hermitian pair.

Lemma 2.3. Let $J_{t}$ be a family of almost complex structures, and let $(\partial / \partial t) J=K$. Then

$$
K J+J K=0 .
$$


Proof. By definition,

$$
0=\frac{\partial}{\partial t} J^{2}=K J+J K
$$

Lemma 2.4. Let $(g, J)$ be an almost Hermitian structure, $K \in \Gamma(\operatorname{End}(T M))$. Then

$$
K J+J K=0 \quad \Longleftrightarrow \quad K \text { is }(0,2)+(2,0) .
$$

Proof. By definition,

$$
\langle(K J+J K) X, Y\rangle=K(J X, Y)-K(X, J Y)=2 K^{(1,1)}(J X, Y) .
$$

Remark 2.5. Similarly, $K J=J K$ if and only if $K$ is $(1,1)$.

Lemma 2.6. Let $J_{t}$ be a family of almost complex structures, and let $(\partial / \partial t) J=K$. Let $g_{t}$ be a family of Riemannian structures compatible with $J_{t}$, and let $(\partial / \partial t) g=h$. Then

$$
K^{\mathrm{sym}} J=h^{(0,2)+(2,0)} .
$$

Proof. By using the equation $K J+J K=0$, we have

$$
\begin{aligned}
0 & =\frac{\partial}{\partial t}(g(J X, J Y)-g(X, Y)) \\
& =h(J X, J Y)-h(X, Y)+g(K X, J Y)+g(J X, K Y) \\
& =-2 h^{(0,2)+(2,0)}(X, Y)+K(J X, Y)+K(Y, J X) \\
& =-2 h^{(0,2)+(2,0)}(X, Y)+2\left(K^{\mathrm{sym}} J\right)(X, Y) .
\end{aligned}
$$

Lemma 2.7. Let $(g, J)$ be an almost Hermitian structure. Then $\left(L_{X} g, L_{X} J\right)$ satisfies the necessary condition of a variation of $(g, J)$, i.e.,

(i) $L_{X} g$ is symmetric,

(ii) $L_{X} J$ is $(0,2)+(2,0)$,

(iii) $\left(L_{X} J\right)^{\mathrm{sym}} J=\left(L_{X} g\right)^{(0,2)+(2,0)}$.

Proof. Let $\phi_{t}$ be the 1-parameter transformation groups generated by $X$, and let $g_{t}=\phi_{t}^{*} g$ and $J_{t}=\phi_{t}^{*} J$. Then

$$
\left.\frac{\partial}{\partial t}\right|_{t=0} g_{t}=L_{X} g,\left.\quad \frac{\partial}{\partial t}\right|_{t=0} J_{t}=L_{X} J
$$

Then Lemma 2.7 follows from Lemmas 2.3, 2.4 and 2.6.

Lemma 2.8. Let $(g, J)$ be an almost Hermitian structure. Then

$$
\begin{aligned}
\left\langle\left(D_{X} J\right) Y, Z\right\rangle & =-\left\langle\left(D_{X} J\right) Z, Y\right\rangle, \\
\left(D_{X} J\right) J Y & =-J\left(D_{X} J\right) Y .
\end{aligned}
$$


Proof. Let $X, Y, Z$ be in a normal coordinate system. Then

$$
\left\langle\left(D_{X} J\right) Y, Z\right\rangle=\left\langle D_{X}(J Y), Z\right\rangle=X\langle J Y, Z\rangle=-X\langle Y, J Z\rangle=-\left\langle\left(D_{X} J\right) Z, Y\right\rangle,
$$

and

$$
\left(D_{X} J\right) J Y=D_{X}(J J Y)-J D_{X}(J Y)=-J\left(D_{X} J\right) Y .
$$

Lemma 2.9 [Gauduchon 1997]. Let $(g, J)$ be an almost Hermitian structure. Then

$$
\begin{aligned}
& \left\langle\left(D_{J X} J\right) Y, Z\right\rangle-\left\langle J\left(D_{X} J\right) Y, Z\right\rangle=\frac{1}{2}(N(X, Y, Z)+N(Z, X, Y)-N(Y, Z, X)), \\
& \left\langle\left(D_{J X} J\right) Y, Z\right\rangle+\left\langle J\left(D_{X} J\right) Y, Z\right\rangle=(d \omega)^{+}(J X, Y, Z)-(d \omega)^{+}(J X, J Y, J Z) .
\end{aligned}
$$

In particular,

$$
\begin{aligned}
D_{J X} J=J D_{X} J & \Longleftrightarrow N=0, \\
D_{J X} J=-J D_{X} J & \Longleftrightarrow(d \omega)^{+}=0 .
\end{aligned}
$$

\section{Main calculations}

First, we define the tensors we use in this paper.

Definition 3.1. Let $(M, g, J)$ be an almost Hermitian manifold, $X, Y, Z \in T M$.

- $B^{1}(X, Y)=\left\langle\left(D_{X} J\right) i,\left(D_{Y} J\right) i\right\rangle$,

- $B^{2}(X, Y)=\left\langle\left(D_{i} J\right) X,\left(D_{i} J\right) Y\right\rangle$,

- $B^{3}(X, Y)=\left\langle\left(D_{\left(D_{i} J\right) X} J\right) i, Y\right\rangle=-\left\langle\left(D_{i} J\right) X, j\right\rangle\left\langle\left(D_{j} J\right) Y, i\right\rangle$,

- $B^{4}(X, Y)=\left\langle\left(D_{X} J\right) i,\left(D_{i} J\right) Y\right\rangle$,

- $\overline{B^{1}}(X, Y)=\left\langle\left(D_{X} J\right) i,\left(D_{Y} J\right) J i\right\rangle$,

- $\overline{B^{2}}(X, Y)=\left\langle\left(D_{i} J\right) X,\left(D_{J i} J\right) Y\right\rangle$,

- $Q_{1}=-\frac{1}{2}\left(B^{1}\right)^{(1,1)}-\left(B^{3}\right)^{(0,2)+(2,0)}+4\left(B^{4}\right)^{(1,1), \text { sym }}-\left(\overline{B^{1}} J\right)^{(1,1)}-\overline{B^{2}} J$,

- $Q_{2}=\left(B^{3}\right)^{(0,2)+(2,0)} J$,

- $\mathcal{N}=B^{2} J$,

- $\mathscr{R}(X, Y)=\operatorname{Ric}(J X, Y)+\operatorname{Ric}(X, J Y)$,

- $2=B^{2} J+B^{3} J$,

- $H(X, Y, Z)=d^{c} \omega(X, Y, Z)=-d \omega(J X, J Y, J Z)$,

- $\mathscr{B}(X, Y)=H(X, i, j) H(Y, i, j)$,

- $\theta^{\sharp}=-J\left(D_{i} J\right) i$,

- $\bar{N}(X, Y)=\frac{1}{2}\left(N\left(\left(D_{i} J\right) X, i, Y\right)+N\left(Y,\left(D_{i} J\right) X, i\right)-N\left(i, Y,\left(D_{i} J\right) X\right)\right)-$ $\frac{1}{2}\left(N\left(i,\left(D_{X} J\right) i, Y\right)+N\left(Y, i,\left(D_{X} J\right) i\right)-N\left(\left(D_{X} J\right) i, Y, i\right)\right)-\left(D_{i} J\right) N(X, i)$,

- $\mathscr{K}(X)=\left(D_{i} N\right)(J i, X)$, 
- $(d \omega)^{+}(X, Y, Z)=\frac{1}{4}(3 d \omega(X, Y, Z)+d \omega(J X, J Y, Z)+d \omega(J X, Y, J Z)+$ $d \omega(X, J Y, J Z))$.

The lemmas below are preparation for the proof of Theorem 1.1.

Lemma 3.2. Let $(g, J)$ be an almost Hermitian structure. Then $\left(-2 \mathrm{Ric}+Q_{1}\right.$, $\triangle J+\mathcal{N}+\mathscr{R}+Q_{2}$ ) satisfies the necessary condition of a variation.

Proof. First, we show that ( -2 Ric, $\triangle J+\mathcal{N}+\mathscr{R})$ satisfies the necessary condition. We need to check the following things:

(i) Ric is symmetric,

(ii) $\triangle J+\mathcal{N}$ is $(0,2)+(2,0)$,

(iii) $\mathscr{R}$ is $(0,2)+(2,0)$,

(iv) $\triangle J$ is skew,

(v) $\mathcal{N}$ is skew,

(vi) $\mathscr{R}$ is symmetric,

(vii) $\mathscr{R} J=-2 \operatorname{Ric}^{(0,2)+(2,0)}$.

By definition, it is easy to see (i), (iii), (vi), (vii). For (ii), we use normal coordinates to calculate the $(1,1)$ part of $\triangle J$, by using Lemma 2.8 :

$$
\begin{aligned}
\langle(\triangle J)(J X), J Y\rangle & =\left\langle\left(D_{i} D J\right)(i, J X), J Y\right\rangle \\
& =\left\langle D_{i}\left(\left(D_{i} J\right)(J X)\right)-\left(D_{i} J\right)\left(D_{i}(J X)\right), J Y\right\rangle \\
& =-\left\langle D_{i}\left(J\left(D_{i} J\right) X\right)+\left(D_{i} J\right)\left(D_{i}(J X)\right), J Y\right\rangle \\
& =-\left\langle\left(D_{i} J\right)\left(D_{i} J\right) X+J D_{i}\left(\left(D_{i} J\right) X\right)+\left(D_{i} J\right)\left(D_{i} J\right) X, J Y\right\rangle \\
& =-2\left\langle\left(D_{i} J\right)(J X),\left(D_{i} J\right) Y\right\rangle-\left\langle\left(D_{i} D_{i} J\right) X, Y\right\rangle \\
& =-2 \mathcal{N}-\langle(\triangle J) X, Y\rangle .
\end{aligned}
$$

So $\mathcal{N}=-(\triangle J)^{(1,1)}$. For (iv), we also use normal coordinates:

$$
\begin{aligned}
\langle(\triangle J) X, Y\rangle & =\left\langle D_{i}\left(\left(D_{i} J\right) X\right), Y\right\rangle \\
& =\partial_{i}\left\langle\left(D_{i} J\right) X, Y\right\rangle \\
& =\partial_{i}\left\langle D_{i}(J X), Y\right\rangle-\partial_{i}\left\langle J\left(D_{i} X\right), Y\right\rangle \\
& =\partial_{i} \partial_{i}\langle J X, Y\rangle-\partial_{i}\left\langle J X, D_{i} Y\right\rangle+\partial_{i}\left\langle D_{i} X, J Y\right\rangle,
\end{aligned}
$$

so we see that $\triangle J$ is skew. And (v) follows from Lemma 2.8.

Next, we show that $\left(Q_{1}, Q_{2}\right)$ satisfies the necessary condition. In fact, by applying Lemma 2.8, we can easily obtain that all terms in $Q_{1}$ are symmetric and all terms in $Q_{2}$ are $(0,2)+(2,0)$. And $Q_{1}^{(0,2)+(2,0)}=\left(B^{3}\right)^{(0,2)+(2,0)}$. This completes the proof. 
Lemma 3.3. Let $(g, J)$ be an almost Hermitian structure. Suppose $d \omega=0$. Then

$$
\begin{aligned}
& Q_{1}=\frac{1}{2} B^{1}-B^{2}, \\
& Q_{2}=0 .
\end{aligned}
$$

Proof. Since $d \omega=0$, by Lemma 2.8 and Lemma 2.9 , one sees that $B^{1}$ and $B^{3}$ are $(1,1)$, that $\overline{B^{1}} J=B^{1}$, and that $\overline{B^{2}} J=B^{2}$. Now we prove that $B^{4}=\frac{1}{2} B^{1}$. In fact, we notice that

$$
\left\langle\left(D_{X} J\right) Y, Z\right\rangle+\left\langle\left(D_{Y} J\right) Z, X\right\rangle+\left\langle\left(D_{Z} J\right) X, Y\right\rangle=d \omega(X, Y, Z)=0 .
$$

Thus,

$$
\begin{aligned}
\left\langle\left(D_{X} J\right) i,\left(D_{i} J\right) Y\right\rangle & =\left\langle\left(D_{i} J\right) Y, j\right\rangle\left\langle\left(D_{X} J\right) i, j\right\rangle \\
& =-\left\langle\left(D_{\left(D_{X} J\right) i} J\right) Y, i\right\rangle \\
& =\left\langle\left(D_{Y} J\right) i,\left(D_{X} J\right) i\right\rangle+\left\langle\left(D_{i} J\right)\left(D_{X} J\right) i, Y\right\rangle \\
& =B^{1}(X, Y)-\left\langle\left(D_{X} J\right) i,\left(D_{i} J\right) Y\right\rangle .
\end{aligned}
$$

So $\left\langle\left(D_{i} J\right) X,\left(D_{Y} J\right) i\right\rangle=\frac{1}{2} B^{1}(X, Y)$. This completes the proof.

Lemma 3.4. Let $(g, J)$ be an almost Hermitian structure. Suppose $N=0$. Then

$$
\begin{aligned}
Q_{1} & =\frac{1}{2} \mathscr{B}, \\
Q_{2} & =2-\mathcal{N} .
\end{aligned}
$$

Proof. The proof is by direct calculations based on Lemma 2.8 and Lemma 2.9. We notice that $B^{1}$ is $(1,1)$ and that $B^{3}$ is $(0,2)+(2,0)$. And $\overline{B^{1}}=B^{1} J$ and $\overline{B^{2}}=B^{2} J$. We also have $B^{4}=0$, since

$$
\begin{aligned}
\left\langle\left(D_{X} J\right) i,\left(D_{i} J\right) Y\right\rangle & =\left\langle\left(D_{X} J\right) J i,\left(D_{J i} J\right) Y\right\rangle \\
& =-\left\langle J\left(D_{X} J\right) i, J\left(D_{i} J\right) Y\right\rangle \\
& =-\left\langle\left(D_{X} J\right) i,\left(D_{i} J\right) Y\right\rangle .
\end{aligned}
$$

We can calculate $\mathscr{B}$ in terms of $D J$ :

$$
\begin{aligned}
\mathscr{B}(X, Y) & =H(X, i, j) H(Y, i, j) \\
& =d \omega(J X, J i, J j) d \omega(J Y, J i, J j) \\
& =d \omega(J X, i, j) d \omega(J Y, i, j) .
\end{aligned}
$$

We have

$$
d \omega(J X, i, j)=\left\langle\left(D_{J X} J\right) i, j\right\rangle+\left\langle\left(D_{J i} J\right) j, X\right\rangle+\left\langle\left(D_{J j} J\right) X, i\right\rangle .
$$


Calculating term by term,

$$
\begin{aligned}
\left\langle\left(D_{J X} J\right) i, j\right\rangle\left\langle\left(D_{J Y} J\right) i, j\right\rangle & =\left\langle\left(D_{X} J\right) i,\left(D_{Y} J\right) i\right\rangle=B^{1}(X, Y), \\
\left\langle\left(D_{J i} J\right) j, X\right\rangle\left\langle\left(D_{J i} J\right) j, Y\right\rangle & =\left\langle\left(D_{J j} J\right) X, i\right\rangle\left\langle\left(D_{J j} J\right) Y, i\right\rangle=\left\langle\left(D_{i} J\right) X,\left(D_{i} J\right) Y\right\rangle \\
& =B^{2}(X, Y), \\
\left\langle\left(D_{J X} J\right) i, j\right\rangle\left\langle\left(D_{J i} J\right) j, Y\right\rangle & =\left\langle\left(D_{J X} J\right) i, j\right\rangle\left\langle\left(D_{J j} J\right) Y, i\right\rangle \\
& =-\left\langle\left(D_{X} J\right) i,\left(D_{i} J\right) Y\right\rangle=0, \\
\left\langle\left(D_{J Y} J\right) i, j\right\rangle\left\langle\left(D_{J i} J\right) j, X\right\rangle & =\left\langle\left(D_{J Y} J\right) i, j\right\rangle\left\langle\left(D_{J j} J\right) X, i\right\rangle \\
& =-\left\langle\left(D_{X} J\right) i,\left(D_{i} J\right) Y\right\rangle=0, \\
\left\langle\left(D_{J i} J\right) j, X\right\rangle\left\langle\left(D_{J j} J\right) Y, i\right\rangle & =\left\langle\left(D_{J i} J\right) j, Y\right\rangle\left\langle\left(D_{J j} J\right) X, i\right\rangle \\
& =-\left\langle\left(D_{\left(D_{i} J\right) X} J\right) i, Y\right\rangle=-B^{3}(X, Y) .
\end{aligned}
$$

So

$$
\frac{1}{2} \mathscr{B}=\frac{1}{2} B^{1}+B^{2}-B^{3} .
$$

Then we obtain the desired result.

Remark 3.5. In [Streets and Tian 2012], 2 is defined as

$$
\begin{aligned}
\mathscr{2}(X)=- & \left(D_{i} J\right)\left(D_{J X} J\right) i-J\left(D_{\left(D_{i} J\right) X} J\right) i+\left(D_{i} J\right)\left(D_{J i} J\right) X \\
& -\left(D_{J\left(D_{i} J\right) i} J\right) X+J\left(D_{\left(D_{i} J\right) i} J\right) X+\left(D_{J X} J\right)\left(D_{i} J\right) i-J\left(D_{X} J\right)\left(D_{i} J\right) i .
\end{aligned}
$$

Since $N=0$, it coincides with our definition.

Lemma 3.6. Let $(g, J)$ be an almost Hermitian structure. Then

$$
L_{\theta^{\sharp}} J=\triangle J+\mathscr{2}+\mathscr{R}+\mathscr{K}+\bar{N} .
$$

Proof. In [Streets and Tian 2012], there is a similar formula. But in our case we don't assume that $N=0$.

We use normal coordinates:

$$
\begin{aligned}
\left(L_{\theta^{\sharp}} J\right) X= & \left(L_{-J\left(D_{i} J\right) i} J\right) X \\
=- & {\left[J\left(D_{i} J\right) i, J X\right]+J\left[J\left(D_{i} J\right) i, X\right] } \\
=- & D_{J\left(D_{i} J\right) i}(J X)+D_{J X}\left(J\left(D_{i} J\right) i\right) \\
& \quad+J D_{J\left(D_{i} J\right) i} X-J D_{X}\left(J\left(D_{i} J\right) i\right) \\
=- & \left(D_{J\left(D_{i} J\right) i} J\right) X+\left(D_{J X} J\right)\left(D_{i} J\right) i+J D_{J X}\left(\left(D_{i} J\right) i\right) \\
& \quad-J\left(D_{X} J\right)\left(D_{i} J\right) i+D_{X}\left(\left(D_{i} J\right) i\right) \\
=- & \left(D_{J\left(D_{i} J\right) i} J\right) X+\left(D_{J X} J\right)\left(D_{i} J\right) i+J\left(D_{J X}\left(D_{i} J\right)\right) i \\
& \quad-J\left(D_{X} J\right)\left(D_{i} J\right) i+D_{X}\left(D_{i} J\right)
\end{aligned}
$$




$$
\begin{aligned}
=J & \left(D^{2} J\right)(J X, i, i)+\left(D^{2} J\right)(X, i, i)-\left(D_{J\left(D_{i} J\right) i} J\right) X \\
& +\left(D_{J X} J\right)\left(D_{i} J\right) i-J\left(D_{X} J\right)\left(D_{i} J\right) i .
\end{aligned}
$$

By the Ricci identity,

$$
\begin{aligned}
\left(D^{2} J\right)(X, i, i) & =\left(D^{2} J\right)(i, X, i)+(\operatorname{Rm}(X, i) J) i \\
& =\left(D^{2} J\right)(i, X, i)+\operatorname{Rm}(X, i)(J i)-J \operatorname{Rm}(X, i) i \\
& =\left(D^{2} J\right)(i, X, i)+\operatorname{Rm}(X, i)(J i)-J \operatorname{Ric}(X) .
\end{aligned}
$$

Similarly,

(6) $J\left(D^{2} J\right)(J X, i, i)=J\left(D^{2} J\right)(i, J X, i)+J \operatorname{Rm}(J X, i)(J i)+\operatorname{Ric}(J X)$.

Notice that

$$
N(X, Y)=\left(D_{J X} J\right) Y-\left(D_{J Y} J\right) X-J\left(D_{X} J\right) Y+J\left(D_{Y} J\right) X .
$$

Hence,

$$
\begin{aligned}
J\left(D^{2} J\right)(i, J X, i)= & J D_{i}\left(\left(D_{J X} J\right) i\right)-J\left(D_{\left(D_{i} J\right) X} J\right) i \\
& -J D_{i}\left(J\left(D_{X} J\right) i\right)+J D_{i}\left(J\left(D_{X} J\right) i\right) \\
= & J D_{i}\left(\left(D_{J X} J\right) i-J\left(D_{X} J\right) i\right)-J\left(D_{\left(D_{i} J\right) X} J\right) i \\
& \quad+J\left(D_{i} J\right)\left(D_{X} J\right) i-\left(D^{2} J\right)(i, X, i) \\
= & J D_{i}\left(\left(D_{J i} J\right) X-J\left(D_{i} J\right) X\right)+J D_{i}(N(X, i)) \\
& -J\left(D_{\left(D_{i} J\right) X} J\right) i+J\left(D_{i} J\right)\left(D_{X} J\right) i-\left(D^{2} J\right)(i, X, i)
\end{aligned}
$$

Notice that

$$
\begin{aligned}
J D_{i}(N(X, i)) & =D_{i}(J N(X, i))-\left(D_{i} J\right) N(X, i) \\
& =D_{i}(N(J i, X))-\left(D_{i} J\right) N(X, i) \\
& =\left(D_{i} N\right)(J i, X)+N\left(\left(D_{i} J\right) i, X\right)-\left(D_{i} J\right) N(X, i) .
\end{aligned}
$$

So

$$
\begin{aligned}
& J\left(D^{2} J\right)(i, J X, i)=J\left(D^{2} J\right)(i, J i, X)+J\left(D_{\left(D_{i} J\right) i} J\right) X \\
&-J\left(D_{i} J\right)\left(D_{i} J\right) X+(\triangle J) X+\mathscr{K}(X)+N\left(\left(D_{i} J\right) i, X\right)-\left(D_{i} J\right) N(X, i) \\
&-J\left(D_{\left(D_{i} J\right) X} J\right) i+J\left(D_{i} J\right)\left(D_{X} J\right) i-\left(D^{2} J\right)(i, X, i) .
\end{aligned}
$$

And

$$
\begin{aligned}
N\left(\left(D_{i} J\right) i, X\right)= & \left(D_{J\left(D_{i} J\right) i} J\right) X-\left(D_{J X} J\right)\left(D_{i} J\right) i \\
& +\left(D_{\left(D_{i} J\right) i} J\right) J X-\left(D_{X} J\right) J\left(D_{i} J\right) i \\
= & \left(D_{J\left(D_{i} J\right) i} J\right) X-\left(D_{J X} J\right)\left(D_{i} J\right) i \\
& -J\left(D_{\left(D_{i} J\right) i} J\right) X+J\left(D_{X} J\right)\left(D_{i} J\right) i .
\end{aligned}
$$


By resorting to Lemma 2.9, we obtain

$$
\begin{aligned}
\langle-J & \left.\left(D_{\left(D_{i} J\right) X} J\right) i-\left(D_{\left(D_{i} J\right) J X} J\right) i, Y\right\rangle \\
& =\left\langle-J\left(D_{\left(D_{i} J\right) X} J\right) i+\left(D_{J\left(D_{i} J\right) X} J\right) i, Y\right\rangle \\
& =\frac{1}{2}\left(N\left(\left(D_{i} J\right) X, i, Y\right)+N\left(Y,\left(D_{i} J\right) X, i\right)-N\left(i, Y,\left(D_{i} J\right) X\right)\right),
\end{aligned}
$$

and

(10) $\quad\left\langle J\left(D_{i} J\right)\left(D_{X} J\right) i, Y\right\rangle$

$$
\begin{aligned}
& =\left\langle J\left(D_{i} J\right)\left(D_{X} J\right) i-\left(D_{J i} J\right)\left(D_{X} J\right) i, Y\right\rangle \\
& =-\frac{1}{2}\left(N\left(i,\left(D_{X} J\right) i, Y\right)+N\left(Y . i,\left(D_{X} J\right) i\right)-N\left(\left(D_{X} J\right) i, Y, i\right)\right) .
\end{aligned}
$$

Then, by the Ricci identity again,

$$
\begin{aligned}
J D^{2} J(i, J i, X) & =\frac{1}{2}\left(J D^{2} J(i, J i, X)-J D^{2} J(J i, i, X)\right) \\
& =\frac{1}{2} J(\operatorname{Rm}(i, J i) J) X \\
& =\frac{1}{2}(J \operatorname{Rm}(i, J i)(J X)+\operatorname{Rm}(i, J i) X) .
\end{aligned}
$$

By the Bianchi identity,

$$
\mathrm{Rm}(i, J i)(J X)+\mathrm{Rm}(J i, J X) i+\operatorname{Rm}(J X, i)(J i)=0 .
$$

Notice that

$$
\operatorname{Rm}(J i, J X) i=\operatorname{Rm}(J X, i)(J i) .
$$

Thus

$$
\begin{gathered}
J \operatorname{Rm}(i, J i)(J X)=-2 J \operatorname{Rm}(J X, i)(J i), \\
\operatorname{Rm}(i, J i)(X)=-2 \operatorname{Rm}(X, i)(J i) .
\end{gathered}
$$

Putting (4)-(13) together, we obtain the desired result.

\section{Proof of Theorem 1.1}

The argument is the same as in [Streets and Tian 2014]. We use DeTurck trick to prove short-time existence and uniqueness.

We consider the following equations:

$$
\begin{aligned}
\frac{\partial}{\partial t} g & =-2 \operatorname{Ric}+Q_{1}+L_{X} g \triangleq \mathscr{D}_{1}(g, J), \\
\frac{\partial}{\partial t} J & =\triangle J+\mathcal{N}+\mathscr{R}+Q_{2}+L_{X} J \triangleq \mathscr{D}_{2}(g, J), \\
g(0) & =g_{0}, \\
J(0) & =J_{0},
\end{aligned}
$$


where $X=\operatorname{tr}_{g}(\Gamma-\bar{\Gamma})$ and $\bar{\Gamma}$ is the Christoffel symbol of a fixed metric $\bar{g}$.

Then, in order to use the PDE theory in Banach space, we consider the tangent space at $J_{0}$. Denote by $T \mathscr{F}_{J}$ the tangent space at $J$, i.e.,

$$
T \mathscr{g}_{J}=\{E \in \operatorname{End}(T M) \mid E J+J E\} .
$$

Then, in a neighborhood $U$ of $J_{0}$, we can identify $J$ and $E$ by using the map

$$
\pi: T \mathscr{F}_{J_{0}} \supset U^{\prime} \rightarrow U, \quad \pi E=-J_{0} e^{J_{0} E},
$$

and note that $\left.D \pi\right|_{0}=\mathrm{Id}$.

Notice that we don't assume that $(g, J)$ is compatible. So we need to make some modifications. For convenience, we write $g^{J}$ and $g^{-J}$ instead of $g^{(1,1)}$ and $g^{(0,2)+(2,0)}$, respectively, and we do similar things for other tensors. Note that $g^{J}$ is compatible with $J$. We consider the following equations:

$$
\begin{aligned}
\frac{\partial}{\partial t} g & =\mathscr{D}_{1}\left(g^{\pi E}, \pi E\right)+\triangle_{g_{0}}\left(g^{-\pi E}\right) \triangleq \widetilde{\mathscr{D}}_{1}(g, E), \\
\frac{\partial}{\partial t} E & =\left(\left.D \pi\right|_{\pi E}\right)^{-1} \mathscr{D}_{2}\left(g^{\pi E}, \pi E\right) \triangleq \widetilde{\mathscr{D}}_{2}(g, E), \\
g(0) & =g_{0}, \\
E(0) & =0 .
\end{aligned}
$$

Note that $\widetilde{\mathscr{D}}_{1}$ is symmetric, and $\widetilde{\mathscr{D}}_{2}$ is well-defined since $\Delta J+\mathcal{N}+\mathscr{R}+Q_{2}+$ $L_{X} J$ is $(0,2)+(2,0)$ for the pair $\left(g^{J}, J\right)$. So $\widetilde{\mathscr{D}}_{1} \oplus \widetilde{\mathscr{D}}_{2}$ gives an operator from $\Gamma\left(\left(T^{*} M \otimes^{\text {sym }} T^{*} M\right) \oplus T \mathscr{F}_{J_{0}}\right)$ to itself.

Now, we calculate the symbol of $\widetilde{D}_{1} \oplus \widetilde{\mathscr{D}}_{2}$ at $\left(g_{0}, 0\right)$ to show the short-time existence of the modified flow. First, we calculate the variation of $\widetilde{\mathscr{D}}_{1}$ along the direction of $(h, 0)$, where $h=\delta g$. Since $\delta E=0, \pi E=\pi 0=J_{0}$ is fixed. And note that $\delta\left(g^{J_{0}}\right)=h^{J_{0}}$ and $g_{0}^{J_{0}}=g_{0}$. Therefore

$$
\mathscr{L}_{\left(g_{0}, 0\right)}\left(\mathscr{D}_{1}\left(g^{\pi E}, \pi E\right)\right)(h, 0)=\mathscr{L}_{g_{0}}\left(\mathscr{D}_{1}\left(g, J_{0}\right)\right)\left(h^{J_{0}}\right)=\mathscr{L}_{g_{0}}\left(\mathscr{D}_{1}\left(g, J_{0}\right)\right)\left(h^{J_{0}}\right),
$$

where $\mathscr{L}_{\left(g_{0}, 0\right)}$ denotes the linearization operator at $\left(g_{0}, 0\right)$.

Noting that only -2 Ric and $L_{X} g$ involve second-order terms, and from standard calculations in Ricci flow [Chow and Knopf 2004] we have

$$
\mathscr{L}_{g_{0}}\left(\mathscr{D}_{1}\left(g, J_{0}\right)\right)\left(h^{J_{0}}\right)=\triangle_{g_{0}}\left(h^{J_{0}}\right)+\mathscr{O}(\partial h) .
$$

And

$$
\mathscr{L}_{\left(g_{0}, 0\right)}\left(\triangle_{g_{0}}\left(g^{-J}\right)\right)(h, 0)=\triangle_{g_{0}}\left(h^{-J_{0}}\right) .
$$

Let $\sigma$ denote the symbol of a linear differential operator. Thus we obtain

$$
\sigma\left(\mathscr{L}_{\left(g_{0}, 0\right)} \widetilde{\mathscr{D}}_{1}\right)(h, 0)(x, \xi)=|\xi|^{2} h, \quad \text { where } \xi \in T_{x}^{*} M .
$$


Then we calculate the variation of $\widetilde{D}_{1}$ along the direction of $(0, K)$, where $K=\delta E$. Since $\left.D \pi\right|_{0}=\mathrm{Id}$, we have

$$
\delta\left(\widetilde{D}_{1}(g, E)\right)(0, K)=\delta\left(\mathscr{D}_{1}\left(g^{J}, J\right)\right)(0, \delta J) .
$$

We identify $\delta J$ and $K$ below.

From the calculations above, we see that

$$
\left(-2 \operatorname{Ric}\left(g^{J}\right)+L_{X\left(g^{J}\right)}\left(g^{J}\right)\right)_{i j}=\left(g^{J}\right)^{p q} \partial_{p} \partial_{q}\left(g^{J}\right)_{i j}+\mathcal{O}(\partial g, \partial J) .
$$

So

$$
\mathscr{L}_{\left(g_{0}, 0\right)}\left(\mathscr{D}_{1}\left(g^{\pi E}, \pi E\right)\right)(0, K)=\left.\frac{\partial}{\partial t}\right|_{t=0}\left(g_{0}\right)^{p q} \partial_{p} \partial_{q}\left(g_{0}^{J_{t}}\right)_{i j}+\mathcal{O}(\partial K) .
$$

It is easy to see that

$$
\mathscr{L}_{\left(g_{0}, 0\right)}\left(\triangle_{g_{0}}\left(g^{-J}\right)\right)(0, K)=\left.\frac{\partial}{\partial t}\right|_{t=0}\left(g_{0}\right)^{p q} \partial_{p} \partial_{q}\left(g_{0}^{-J_{t}}\right)_{i j}+\mathcal{O}(\partial K) .
$$

Thus we obtain

$$
\sigma\left(\mathscr{L}_{\left(g_{0}, 0\right)} \widetilde{\mathscr{D}}_{1}\right)(0, K)(x, \xi)=0, \quad \text { where } \xi \in T_{x}^{*} M .
$$

Next, we calculate the variation of $\widetilde{\mathscr{D}}_{2}$ along the direction of $(\delta g, \delta E)=(h, K)$. We have

$$
\delta\left(\widetilde{D}_{2}(g, E)\right)(h, K)=\delta\left(\mathscr{D}_{2}\left(g^{J}, J\right)\right)(\delta g, \delta J) .
$$

In the expression for $\mathscr{D}_{2}$, only $\triangle J, L_{X} J$, and $\mathscr{R}$ involve second-order terms, so we only need to calculate these three terms. We calculate them for the pair $(g, J)$ first.

For $\triangle J$, we have

$$
\begin{aligned}
(\triangle J)\left(e_{k}\right) & =g^{i j} D^{2} J\left(e_{i}, e_{j}, e_{k}\right) \\
& =g^{i j} D_{i}\left(\left(D_{j} J\right) e_{k}\right)+\mathcal{O}(\partial g, \partial J) \\
& =g^{i j} D_{i}\left(D_{j}\left(J e_{k}\right)-J D_{j} e_{k}\right)+\mathcal{O}(\partial g, \partial J) \\
& =g^{i j} D_{i}\left(D_{j}\left(J_{k}^{l} e_{l}\right)-J\left(\Gamma_{j k}^{p} e_{p}\right)\right)+\mathcal{O}(\partial g, \partial J) \\
& =g^{i j}\left(D_{i}\left(\partial_{j} J_{k}^{l} e_{l}\right)+D_{i}\left(J_{k}^{p} \Gamma_{j p}^{l} e_{l}\right)-D_{i}\left(\Gamma_{j k}^{p} J_{p}^{l} e_{l}\right)\right)+\mathcal{O}(\partial g, \partial J) \\
& =g^{i j}\left(\partial_{i} \partial_{j} J_{k}^{l}+J_{k}^{p} \partial_{i} \Gamma_{j p}^{l}-J_{p}^{l} \partial_{i} \Gamma_{j k}^{p}\right) e_{l}+\mathcal{O}(\partial g, \partial J) .
\end{aligned}
$$

For $L_{X} J$, we have

$$
\begin{aligned}
\left(L_{X} J\right)\left(e_{k}\right) & =\left[X, J e_{k}\right]-J\left[X, e_{k}\right] \\
& =\left[X^{p} e_{p}, J_{k}^{l} e_{l}\right]-J\left[X^{p} e_{p}, e_{k}\right] \\
& =\left(X^{p} \partial_{p} J_{k}^{l}-J_{k}^{p} \partial_{p} X^{l}+J_{p}^{l} \partial_{k} X^{p}\right) e_{l} \\
& =g^{i j}\left(J_{p}^{l} \partial_{k} \Gamma_{i j}^{p}-J_{k}^{p} \partial_{p} \Gamma_{i j}^{l}\right) e_{l}+O(\partial g, \partial J) .
\end{aligned}
$$


For $R$, we have

$$
\begin{aligned}
\mathscr{R}\left(e_{k}\right) & =\left(J_{k}^{p} \operatorname{Ric}_{p}^{l}-J_{p}^{l} \operatorname{Ric}_{k}^{p}\right) e_{l} \\
& =g^{i j}\left(-J_{k}^{p} \partial_{i} \Gamma_{p j}^{l}+J_{k}^{p} \partial_{p} \Gamma_{i j}^{l}+J_{p}^{l} \partial_{i} \Gamma_{k j}^{p}-J_{p}^{l} \partial_{k} \Gamma_{i j}^{p}\right) e_{l}+\mathcal{O}(\partial g, \partial J) .
\end{aligned}
$$

So we obtain

$$
\left(\triangle J+\mathscr{R}+L_{X} J\right)_{k}^{l}=g^{i j} \partial_{i} \partial_{j} J_{k}^{l}+\mathcal{O}(\partial g, \partial J) .
$$

As for the pair $\left(g^{J}, J\right)$, the lower-order terms are still lower-order terms, and when we evaluate at $\left(g_{0}, J_{0}\right)$, from the compatibility, we have

$$
\left(\mathscr{L}_{\left(g_{0}, 0\right)} \widetilde{\mathscr{D}}_{2}\right)(h, K)=\triangle_{g_{0}} K+\mathcal{O}(\partial g, \partial J) .
$$

Hence, the total symbol is

$$
\sigma\left(\mathscr{L}_{\left(g_{0}, 0\right)} \widetilde{\mathscr{D}}\right)(h, K)(x, \xi)=\left(\begin{array}{cc}
|\xi|^{2} & 0 \\
0 & |\xi|^{2}
\end{array}\right) .
$$

By the standard theory of parabolic PDE, there exists a unique short-time solution of (15).

Next we show that, under (15), $(g, J)$ is compatible, where $J=\pi E$. Suppose that $(g, J)$ exists for $t \in\left[0, \epsilon_{0}\right]$. Then by the compactness of $M$, in this time interval, every tensor we involve is bounded. Let $(\partial / \partial t) J=K$. Then

$$
\begin{aligned}
\frac{\partial}{\partial t}\left|g^{-J}\right|_{g^{J}}^{2}= & 2\left\langle\frac{\partial}{\partial t}\left(g^{-J}\right), g^{-J}\right\rangle_{g^{J}}+C *\left(g^{-J}\right)^{* 2} \\
= & 2\left\langle\frac{\partial}{\partial t} \frac{1}{2}(g(\cdot, \cdot)-g(J \cdot, J \cdot)), g^{-J}\right\rangle_{g^{J}}+C *\left(g^{-J}\right)^{* 2} \\
= & 2\left\langle\left(\frac{\partial}{\partial t} g\right)^{-J}, g^{-J}\right\rangle_{g^{J}}-\left\langle g(J \cdot, K \cdot)+g(K \cdot, J \cdot), g^{-J}\right\rangle_{g^{J}}+C *\left(g^{-J}\right)^{* 2} \\
\leq & \left\langle 2\left(\mathscr{D}_{1}\left(g^{J}, J\right)\right)^{-J}+2\left(\triangle_{g_{0}}\left(g^{-J}\right)\right)^{-J}-g(J \cdot, K \cdot)-g(K \cdot, J \cdot), g^{-J}\right\rangle_{g^{J}} \\
& \quad+C\left|g^{-J}\right|_{g^{J}}^{2} .
\end{aligned}
$$

Note that $\left(g^{J}, J\right)$ is compatible and $K=\mathscr{D}_{2}\left(g^{J}, J\right)$, so by Lemmas 3.2 and 2.7,

$$
\mathscr{D}_{1}\left(g^{J}, J\right)^{-J}-\frac{1}{2}\left(g^{J}(J \cdot, K \cdot)+g^{J}(K \cdot, J \cdot)\right)=0 .
$$

So

$$
\begin{aligned}
\frac{\partial}{\partial t}\left|g^{-J}\right|_{g^{J}}^{2} & \leq 2\left\langle\left(\triangle_{g_{0}}\left(g^{-J}\right)\right)^{-J}-g^{-J}(J \cdot, K \cdot)-g^{-J}(K \cdot, J \cdot), g^{-J}\right\rangle_{g^{J}}+C\left|g^{-J}\right|_{g^{J}}^{2} \\
& \leq 2\left\langle\left(\triangle_{g_{0}}\left(g^{-J}\right)\right)^{-J}, g^{-J}\right\rangle_{g^{J}}+C\left|g^{-J}\right|_{g^{J}}^{2} .
\end{aligned}
$$

Since $J$ acts isometrically on the space $\Gamma\left(T^{*} M \otimes^{\text {sym }} T^{*} M\right)$ in the induced metric from $g^{J}$, and since the $(1,1)$ tensors and $(0,2)+(2,0)$ tensors correspond to the 
+1 and -1 eigenspaces, respectively, they are orthogonal. So

$$
\left\langle\left(\triangle_{g_{0}}\left(g^{-J}\right)\right)^{J}, g^{-J}\right\rangle_{g^{J}}=0 .
$$

Then,

$$
\frac{\partial}{\partial t}\left|g^{-J}\right|_{g^{J}}^{2} \leq 2\left\langle\triangle_{g_{0}}\left(g^{-J}\right), g^{-J}\right\rangle_{g^{J}}+C\left|g^{-J}\right|_{g^{J}}^{2}
$$

By definition,

$$
\triangle_{g_{0}}\left(g^{-J}\right)=\operatorname{tr}_{g_{0}} D_{g_{0}}^{2}\left(g^{-J}\right) .
$$

Since the second order term about $g^{-J}$ in $D_{g_{0}}^{2}\left(g^{-J}\right)$ is the same as in $D_{g^{J}}^{2}\left(g^{-J}\right)$,

$$
\triangle_{g_{0}}\left(g^{-J}\right)=\operatorname{tr}_{g_{0}}\left(D_{g^{J}}^{2}\left(g^{-J}\right)+C^{\prime} * D_{g^{J}}\left(g^{-J}\right)+C * g^{-J}\right) .
$$

Let $A$ be any tensor. We have the formula

$$
\begin{aligned}
D^{2}\langle A, A\rangle & =D(D\langle A, A\rangle) \\
& =2 D\left(\left\langle D_{i} A, A\right\rangle e^{i}\right) \\
& =2\left\langle D_{i, j}^{2} A, A\right\rangle e^{i} \otimes e^{j}+2\left\langle D_{i} A, D_{j} A\right\rangle e^{i} \otimes e^{j} .
\end{aligned}
$$

Let $A=g^{-J}$ and the metric above be $g^{J}$. Taking the trace of each side with respect to $g_{0}$, we obtain

$$
\begin{aligned}
2\left\langle\operatorname{tr}_{g_{0}} D_{g^{J}}^{2}\left(g^{-J}\right), g^{-J}\right\rangle_{g^{J}} & \\
& =\operatorname{tr}_{g_{0}} D_{g^{J}}^{2}\left(\left|g^{-J}\right|_{g^{J}}^{2}\right)-2\left\langle D_{g^{J}} g^{-J}\left(e_{i}\right), D_{g^{J}} g^{-J}\left(e_{j}\right)\right\rangle_{g^{J}}\left\langle e^{i}, e^{j}\right\rangle_{g_{0}} .
\end{aligned}
$$

Along this flow, for $t \in\left[0, \epsilon_{0}\right], g^{J}$ is uniformly bounded by $g_{0}$, so we have

$$
2\left\langle\operatorname{tr}_{g_{0}} D_{g^{J}}^{2}\left(g^{-J}\right), g^{-J}\right\rangle_{g^{J}} \leq \operatorname{tr}_{g_{0}} D_{g^{J}}^{2}\left(\left|g^{-J}\right|_{g^{J}}^{2}\right)-2 C^{\prime \prime}\left|D_{g^{J}} g^{-J}\right|_{g^{J}}^{2} .
$$

Hence,

$\frac{\partial}{\partial t}\left|g^{-J}\right|_{g^{J}}^{2} \leq \operatorname{tr}_{g_{0}} D_{g^{J}}^{2}\left(\left|g^{-J}\right|_{g^{J}}^{2}\right)-2 C^{\prime \prime}\left|D_{g^{J}} g^{-J}\right|_{g^{J}}^{2}+C^{\prime} * D_{g^{J}}\left(g^{-J}\right) * g^{-J}+C\left|g^{-J}\right|_{g^{J}}^{2}$.

By using the Cauchy inequality on $C^{\prime} * D_{g^{J}}\left(g^{-J}\right) * g^{-J}$, finally we obtain

$$
\frac{\partial}{\partial t}\left|g^{-J}\right|_{g^{J}}^{2} \leq \operatorname{tr}_{g_{0}} D_{g^{J}}^{2}\left(\left|g^{-J}\right|_{g^{J}}^{2}\right)+C\left|g^{-J}\right|_{g^{J}}^{2} .
$$

Notice that $\operatorname{tr}_{g_{0}} D_{g^{J}}^{2}$ is elliptic and $\left|g^{-J}\right|^{2}=0$ at $t=0$. Then by the maximum principle, considering $e^{-C t}\left|g^{-J}\right|^{2}$, we have $\left|g^{-J}\right|^{2}=0$ for $t \in\left[0, \epsilon_{0}\right]$, i.e., $(g, J)$ is compatible. Since $\epsilon_{0}$ is arbitrary, $(g, J)$ is always compatible as long as the solution exists. Because the positivity of $g$ is an open condition, we may assume that $g$ is positive in short time. Then the short-time solution of (15) gives the short-time solution of (14). 
Now, let $(\tilde{g}(t), \tilde{J}(t))$ be a solution of (14) and let $\varphi_{t}$ be the one-parameter family of diffeomorphisms generated by $-X(t)$ defined as above. Let $g(t)=\varphi_{t}^{*} \tilde{g}(t)$, $J(t)=\varphi_{t}^{*} \tilde{J}(t)$. Then

$$
\begin{aligned}
\frac{\partial}{\partial t} g & =\frac{\partial}{\partial t}\left(\varphi_{t}^{*} \tilde{g}(t)\right) \\
& =\varphi_{t}^{*}\left(\frac{\partial}{\partial t} \tilde{g}(t)+L_{(-X(t))} \tilde{g}(t)\right) \\
& =\varphi_{t}^{*}\left(-2 \operatorname{Ric}(\tilde{g}(t))+Q_{1}(\tilde{g}(t))\right) \\
& =-2 \operatorname{Ric}\left(\varphi_{t}^{*} \tilde{g}(t)\right)+Q_{1}\left(\varphi_{t}^{*} \tilde{g}(t)\right) \\
& =-2 \operatorname{Ric}(g)+Q_{1}(g) .
\end{aligned}
$$

So $g(t)$ satisfies the equation. Similarly, $J(t)$ also satisfies the equation. And $(g(t), J(t))$ differs from $(\tilde{g}(t), \tilde{J}(t))$ by a diffeomorphism, so $(g(t), J(t))$ is also an almost Hermitian pair. This completes the existence part of the theorem.

For uniqueness, let $\left(g_{i}, J_{i}\right)$ be two solutions of (3), $i=1,2$. Since $M$ is compact, we can solve the harmonic heat flow

$$
\begin{aligned}
\frac{\partial}{\partial t} \phi_{i}(t) & =\triangle_{g_{i}, \bar{g}} \phi_{i}(t), \\
\phi_{i}(0) & =\mathrm{Id},
\end{aligned}
$$

for $\phi_{i}(t)$ for short time, where $\bar{g}$ is the same fixed metric as above. We can also assume that the $\phi_{i}(t)$ are diffeomorphisms. Let $\hat{g}_{i}=\left(\phi_{i}^{-1}(t)\right)^{*} g_{i}(t)$. Note that

$$
\begin{aligned}
\left(\frac{\partial}{\partial t} \phi_{i}\right)(p) & =\left(\triangle_{g_{i}, \bar{g}} \phi_{i}\right)(p) \\
& =\left(\triangle_{\hat{g}_{i}, \bar{g}} \mathrm{Id}\right)\left(\phi_{i}(p)\right) \\
& =\left(-\hat{g}^{i j}\left(\hat{\Gamma}_{i j}^{k}-\bar{\Gamma}_{i j}^{k}\right) \frac{\partial}{\partial x^{k}}\right)\left(\phi_{i}(p)\right) \\
& =-X_{\hat{g}}\left(\phi_{i}(p)\right) .
\end{aligned}
$$

Then, taking the time derivative of $\left(\phi_{i}(t)\right)^{*} \hat{g}_{i}(t)=g_{i}(t)$, and doing a similar calculation to (16), we see that both $\hat{g}_{i}(t)$ satisfy (14) and they share the same initial data. Since we have proved the compatibility, the symbol of (14) is Id, as we calculated, so the solution of (14) is unique. Then we obtain

$$
\hat{g}_{1}(t)=\hat{g}_{2}(t)=\hat{g}(t), \quad \hat{J}_{1}(t)=\hat{J}_{2}(t)=\hat{J}(t) .
$$

Then from the uniqueness of

$$
\begin{aligned}
\frac{\partial}{\partial t} \phi(t) & =-X_{\hat{g}}(\phi(t)), \\
\phi(0) & =\mathrm{Id},
\end{aligned}
$$


we see the uniqueness of $(g, J)$ for a short while. Then, by continuity, $(g, J)$ is unique as long as it exists.

Next, we check two special cases. Suppose that the initial data is almost Kähler. Then we run the symplectic curvature flow (1). By definitions and Lemma 3.3, we see that, in this situation, $(g, J)$ also satisfies (3). So from the uniqueness of (3), if the initial data is almost Kähler, then (3) coincides with symplectic curvature flow. And a similar argument holds in the pluriclosed case when we apply Lemma 3.4.

Finally, we prove that the flow (3) preserves the integrability of $J$. Let $\left(g_{0}, J_{0}\right)$ be an Hermitian structure. Fix $J_{0}$ and consider the flow

$$
\begin{aligned}
\frac{\partial}{\partial t} \tilde{g} & =-2 \operatorname{Ric}_{\tilde{g}}+Q_{1}\left(\tilde{g}, J_{0}\right)-L_{\theta^{\sharp}\left(\tilde{g}, J_{0}\right)} \tilde{g}, \\
\tilde{g}(0) & =g_{0} .
\end{aligned}
$$

By the DeTurck trick, we see that $\tilde{g}(t)$ exists for a while, but is not necessarily compatible with $J_{0}$ now. Then by a gauge transformation induced by $\theta^{\sharp}\left(\tilde{g}, J_{0}\right)$, we obtain a short-time solution $(g(t), J(t))$ for the flow

$$
\begin{aligned}
\frac{\partial}{\partial t} g & =-2 \operatorname{Ric}_{g}+Q_{1}(g, J), \\
\frac{\partial}{\partial t} J & =L_{\theta^{\sharp}(g, J)} J, \\
g(0) & =g_{0}, \\
J(0) & =J_{0} .
\end{aligned}
$$

We still don't know the compatibility of $(g, J)$ now, but since $J$ is changed just by a diffeomorphism, $N$ always vanishes. By Lemma 2.9, one may write $Q_{2}-2+\mathcal{N}$ in terms of $N$ in the almost Hermitian setting. We denote such a tensor $N_{0}$, i.e., $N_{0}$ is in terms of $N$, and, when $(g, J)$ is compatible, $N_{0}=Q_{2}-2+\mathcal{N}$. So the above flow is the same as the flow

$$
\begin{aligned}
\frac{\partial}{\partial t} g & =-2 \operatorname{Ric}_{g}+Q_{1}(g, J), \\
\frac{\partial}{\partial t} J & =L_{\theta^{\sharp}(g, J)} J+N_{0}(g, J)-\bar{N}(g, J)-\mathscr{K}(g, J), \\
g(0) & =g_{0}, \\
J(0) & =J_{0} .
\end{aligned}
$$

Then by Lemma 3.6, and using the same argument in the proof of short-time existence above, one sees that $(g, J)$ is compatible and coincides with (3), so the integrability of $J$ is preserved.

This completes the proof of Theorem 1.1.

Remark 4.1. Streets and Tian [2014] introduced almost Hermitian curvature flow, where the symbol term deforming $J$ is $-\mathscr{K}$. From Lemma 3.6 we see that, modulo 
lower-order terms, $-\mathscr{K}$ differs from $\triangle J+\mathscr{R}$ just by a gauge term. If we also change the evolution of $g$ by the same gauge transformation, the second derivative of $g$ will appear in $L_{\theta^{\sharp}} g$. So, in general, our flow is not in the family of almost Hermitian curvature flow.

\section{Proof of Theorem 1.2 and Theorem 1.3}

First, we derive the evolution equations of $D J, \mathrm{Rm}$ and their higher covariant derivatives.

Lemma 5.1. Under (3),

$$
\frac{\partial}{\partial t} D J=\triangle D J+\mathrm{Rm} * D J+J^{* 2} * D J^{* 3}+J^{* 3} * D J * D^{2} J .
$$

Proof. Using the fact $\triangle D T-D \triangle T=D \mathrm{Rm} * T+\mathrm{Rm} * D T$, we have

$$
\begin{aligned}
\frac{\partial}{\partial t} D J & =\dot{\Gamma} * J+D \dot{j} \\
& =D\left(\mathrm{Rm}+J^{* 2} * D J^{* 2}\right) * J+D\left(\triangle J+\mathrm{Rm} * J+J * D J^{* 2}\right) \\
& =\triangle D J+D \mathrm{Rm} * J+\mathrm{Rm} * D J+J^{* 2} * D J^{* 3}+J^{* 3} * D J * D^{2} J .
\end{aligned}
$$

Hence we only need to show there is no $D \mathrm{Rm} * J$ term. It is the same calculation as in [Streets and Tian 2014], since the only differences are the first-order terms in $J$, which does not involve a $D \mathrm{Rm}$ term.

Lemma 5.2. Under (3),

$$
\frac{\partial}{\partial t} \mathrm{Rm}=\Delta \mathrm{Rm}+\mathrm{Rm}^{* 2}+\mathrm{Rm} * J^{* 2} * D J^{* 2}+\sum_{\substack{0 \leq k_{1}, \ldots, k_{4} \leq 3 \\ k_{1}+\cdots+k_{4}=4}} D^{k_{1}} J * \cdots * D^{k_{4}} J .
$$

Proof. Let $(\partial / \partial t) g=h$. From the variation formula in Ricci flow (see [Chow and Knopf 2004]) we have

$$
\begin{aligned}
\frac{\partial}{\partial t} \operatorname{Rm}(X, Y, Z, W)=\frac{1}{2} & h(\operatorname{Rm}(X, Y) Z, W)-h(\operatorname{Rm}(X, Y) W, Z)) \\
& +\frac{1}{2}\left(D_{Y, W}^{2} h(X, Z)-D_{X, W}^{2} h(Y, Z)\right. \\
& \left.+D_{X, Z}^{2} h(Y, W)-D_{Y, Z}^{2} h(X, W)\right) .
\end{aligned}
$$

And, when $h=-2$ Ric,

$$
\frac{\partial}{\partial t} \mathrm{Rm}=\triangle \mathrm{Rm}+\mathrm{Rm}^{* 2} .
$$

Notice that, in (3), $h=(\partial / \partial t) g=-2 \mathrm{Ric}+J^{* 2} * D J^{* 2}$, so we obtain the evolution equation of $\mathrm{Rm}$. 
Proposition 5.3. Under (3),

$$
\frac{\partial}{\partial t} D^{k} J=\Delta D^{k} J+\sum_{\substack{l_{1}+\cdots+l_{5}=k+2 \\ 0 \leq l_{1}, \ldots, l_{5} \leq k+1}} D^{l_{1}} J * \cdots * D^{l_{5}} J+\sum_{l=0}^{k-1} D^{l} \mathrm{Rm} * D^{k-l} J
$$

and

$$
\begin{aligned}
\frac{\partial}{\partial t} D^{k} \mathrm{Rm}=\Delta D^{k} \mathrm{Rm} & +\sum_{\substack{l_{1}+\ldots+l_{4}=k+4 \\
0 \leq l_{1}, \ldots, l_{4} \leq k+3}} D^{l_{1}} J * \cdots * D^{l_{4}} J+\sum_{l=0}^{k} D^{l} \mathrm{Rm} * D^{k-l} \mathrm{Rm} \\
& +\sum_{\substack{0 \leq l_{0} \leq k \\
l_{1}+\ldots+l_{4}=k+2-l_{0} \\
0 \leq l_{1}, \ldots, l_{4} \leq k+1}} D^{l_{0}} \mathrm{Rm} * D^{l_{1}} J * \cdots * D^{l_{4}} J .
\end{aligned}
$$

Proof. By using Lemma 5.1 and the fact that $(\partial / \partial t) \Gamma=D\left(\mathrm{Rm}+J^{* 2} * D J^{* 2}\right)$, we have

$$
\begin{aligned}
\frac{\partial}{\partial t} D^{k} J= & \frac{\partial}{\partial t} \Gamma * D^{k-1} J+D \frac{\partial}{\partial t} D^{k-1} J \\
= & \sum_{l=0}^{k-2} D^{l} \frac{\partial}{\partial t} \Gamma * D^{k-1-l} J+D^{k-1} \frac{\partial}{\partial t} D J \\
= & \sum_{l=0}^{k-2} D^{l} D\left(\mathrm{Rm}+J^{* 2} * D J^{* 2}\right) * D^{k-1-l} J \\
& \quad+D^{k-1}\left(\triangle D J+\mathrm{Rm} * D J+J^{* 2} * D J^{* 3}+J^{* 3} * D J * D^{2} J\right) .
\end{aligned}
$$

Interchanging $D$ and $\Delta$, we observe that the highest order of $\mathrm{Rm}$ is $k-1$, and the highest order of $J$ is $k+1$ if not involving $\mathrm{Rm}$. Then we obtain the evolution equation of $D^{k} J$.

As for the evolution equation of $D^{k} \mathrm{Rm}$, the calculation is similar. The key point is to observe the highest order.

Now we can use Proposition 5.3 to prove Theorem 1.2 and Theorem 1.3.

Proof of Theorem 1.2. The proof is similar to the higher derivative estimates in Ricci flow [Chow and Knopf 2004]. We assume $t\left|D^{2} J\right| \leq C$ first. By induction, we will prove

$$
\left|D^{k} J\right| \leq \frac{C}{t^{k / 2}}, \quad\left|D^{k-2} \mathrm{Rm}\right| \leq \frac{C}{t^{k / 2}} .
$$

$(P)$ holds when $k=2$ from the assumption.

Now we assume $(P)$ holds for $k-1$. Consider

$$
F(t)=t^{k+1}\left(\left|D^{k} J\right|^{2}+\left|D^{k-2} \mathrm{Rm}\right|^{2}\right)+\lambda t^{k}\left(\left|D^{k-1} J\right|^{2}+\left|D^{k-3} \mathrm{Rm}\right|^{2}\right),
$$


where $\lambda$ is a large constant to be determined. We will show that

$$
\frac{\partial}{\partial t} F \leq \triangle F+C
$$

Then, by the maximum principle, $(P)$ holds for $k$. Now we prove (17) by using Proposition 5.3:

$$
\begin{aligned}
& \frac{\partial}{\partial t}\left|D^{k} J\right|^{2} \\
& =\left(\mathrm{Rm}+J^{* 2} * D J^{* 2}\right) * D^{k} J^{* 2}+2\left\langle D^{k} J,\right. \\
& \left.\Delta D^{k} J+\sum_{\substack{l_{1}+\cdots+l_{5}=k+2 \\
0 \leq l_{1}, \ldots, l_{5} \leq k+1}} D^{l_{1}} J * \cdots * D^{l_{5}} J+\sum_{l=0}^{k-1} D^{l} \mathrm{Rm} * D^{k-l} J\right\rangle \\
& =\left(\mathrm{Rm}+J^{* 2} * D J^{* 2}\right) * D^{k} J^{* 2}+\Delta\left|D^{k} J\right|^{2}-2\left|D^{k+1} J\right|^{2} \\
& \quad+D^{k} J *\left(\sum_{\substack{l_{1}+\cdots+l_{5}=k+2 \\
0 \leq l_{1}, \ldots, l_{5} \leq k+1}} D^{l_{1}} J * \cdots * D^{l_{5}} J+\sum_{l=0}^{k-1} D^{l} \mathrm{Rm} * D^{k-l} J\right) \\
& =\Delta\left|D^{k} J\right|^{2}-2\left|D^{k+1} J\right|^{2}+\left(\mathrm{Rm}+J^{* 2} * D J^{* 2}\right) * D^{k} J^{* 2} \\
& +D^{k} J * D^{k+1} J * D J * J^{* 3}+D^{k} J * D^{k} J * D J^{* 2} * J^{* 2} \\
& +D^{k} J * D^{k} J * D^{2} J * J^{* 3}+D^{k} J * \sum_{\substack{l_{1}+\cdots+l_{5}=k+2 \\
0 \leq l_{1}, \ldots, l_{5} \leq k-1}} D^{l_{1}} J * \cdots * D^{l_{5}} J \\
& \quad+D^{k} J * \mathrm{Rm} * D^{k} J+D^{k} J * D^{k-1} \mathrm{Rm} * D J+D^{k} J * D^{k-2} \mathrm{Rm} * D^{2} J \\
& +D^{k} J * \sum_{l=1}^{k-3} D^{l} \mathrm{Rm} * D^{k-l} J . \quad
\end{aligned}
$$

From the assumption,

$$
\begin{aligned}
\frac{\partial}{\partial t}\left|D^{k} J\right|^{2} \leq \triangle\left|D^{k} J\right|^{2}-2\left|D^{k+1} J\right|^{2}+\frac{C}{t}\left|D^{k} J\right|^{2}+\frac{C}{t^{1 / 2}}\left|D^{k} J\right|\left|D^{k+1} J\right| \\
+\frac{C}{t^{(k+2) / 2}}\left|D^{k} J\right|+\frac{C}{t^{1 / 2}}\left|D^{k} J\right|\left|D^{k-1} \mathrm{Rm}\right|+\frac{C}{t}\left|D^{k} J\right|\left|D^{k-2} \mathrm{Rm}\right| .
\end{aligned}
$$

Similarly, we obtain

$$
\begin{aligned}
\frac{\partial}{\partial t}\left|D^{k-2} \mathrm{Rm}\right|^{2} \leq \Delta\left|D^{k-2} \mathrm{Rm}\right|^{2}-2\left|D^{k-1} \mathrm{Rm}\right|^{2}+\frac{C}{t}\left|D^{k-2} \mathrm{Rm}\right|^{2} \\
\quad+\frac{C}{t^{1 / 2}}\left|D^{k-2} \mathrm{Rm}\right|\left|D^{k+1} J\right|+\frac{C}{t^{(k+2) / 2}}\left|D^{k-2} \mathrm{Rm}\right|+\frac{C}{t}\left|D^{k} J\right|\left|D^{k-2} \mathrm{Rm}\right| .
\end{aligned}
$$


Then, by the Cauchy-Schwarz inequality,

$$
\begin{aligned}
& \frac{\partial}{\partial t}\left(t^{k+1}\left(\left|D^{k} J\right|^{2}+\left|D^{k-2} \mathrm{Rm}\right|^{2}\right)\right) \leq \triangle\left(t^{k+1}\left(\left|D^{k} J\right|^{2}+\left|D^{k-2} \mathrm{Rm}\right|^{2}\right)\right) \\
&-t^{k+1}\left(\left|D^{k+1} J\right|^{2}+\left|D^{k-1} \mathrm{Rm}\right|^{2}\right)+C t^{k}\left(\left|D^{k} J\right|^{2}+\left|D^{k-2} \mathrm{Rm}\right|^{2}\right)+C .
\end{aligned}
$$

Replacing $k$ with $k-1$ and using the assumption, we obtain

$$
\begin{aligned}
& \frac{\partial}{\partial t}\left(t^{k}\left(\left|D^{k-1} J\right|^{2}+\left|D^{k-3} \mathrm{Rm}\right|^{2}\right)\right) \\
& \leq \triangle\left(t^{k}\left(\left|D^{k-1} J\right|^{2}+\left|D^{k-3} \mathrm{Rm}\right|^{2}\right)\right)-t^{k}\left(\left|D^{k} J\right|^{2}+\left|D^{k-2} \mathrm{Rm}\right|^{2}\right) \\
& \quad+C t^{k-1}\left(\left|D^{k-1} J\right|^{2}+\left|D^{k-3} \mathrm{Rm}\right|^{2}\right)+C \\
& \leq \triangle\left(t^{k}\left(\left|D^{k-1} J\right|^{2}+\left|D^{k-3} \mathrm{Rm}\right|^{2}\right)\right)-t^{k}\left(\left|D^{k} J\right|^{2}+\left|D^{k-2} \mathrm{Rm}\right|^{2}\right)+C .
\end{aligned}
$$

Then

$$
\begin{aligned}
\frac{\partial F}{\partial t} \leq & \Delta F-t^{k+1}\left(\left|D^{k+1} J\right|^{2}+\left|D^{k-1} \mathrm{Rm}\right|^{2}\right) \\
& +(C-\lambda) t^{k}\left(\left|D^{k} J\right|^{2}+\left|D^{k-2} \mathrm{Rm}\right|^{2}\right)+C \\
\leq & \Delta F+(C-\lambda) t^{k}\left(\left|D^{k} J\right|^{2}+\left|D^{k-2} \mathrm{Rm}\right|^{2}\right)+C .
\end{aligned}
$$

We choose $\lambda=C$, so (17) holds.

Now, we prove that $t\left|D^{2} J\right| \leq C$. For $p \in M$, if $\left|D^{2} J\right|_{p, t} \neq 0$, then similarly, by Proposition 5.3,

$$
\begin{aligned}
\frac{\partial}{\partial t}\left|D^{2} J\right|= & \frac{1}{2\left|D^{2} J\right|} \frac{\partial}{\partial t}\left|D^{2} J\right|^{2} \\
= & \frac{1}{2\left|D^{2} J\right|}\left(\triangle\left|D^{2} J\right|^{2}-2\left|D^{3} J\right|^{2}+D^{2} J^{* 3} * J^{* 3}\right. \\
& \quad+D^{3} J * D^{2} J * D J * J^{* 3}+D^{2} J^{* 2} * D J^{* 2} * J^{* 2} \\
& \left.\quad+D^{2} J * D J^{* 4}+D^{2} J^{* 2} * \mathrm{Rm}+D^{2} J * D J * D \mathrm{Rm}\right) .
\end{aligned}
$$

Notice that, for $\left|D^{2} J\right|_{p, t} \neq 0$,

$$
\triangle\left|D^{2} J\right|^{2}=2\left|D^{2} J\right| \triangle\left|D^{2} J\right|+2|D| D^{2} J||^{2} .
$$

So,

$$
\begin{aligned}
\frac{\partial}{\partial t}\left|D^{2} J\right|=\triangle & \left|D^{2} J\right|+\frac{|D| D^{2} J||^{2}}{\left|D^{2} J\right|}+\frac{1}{2\left|D^{2} J\right|}\left(-2\left|D^{3} J\right|^{2}+D^{2} J^{* 3} * J^{* 3}\right. \\
& +D^{3} J * D^{2} J * D J * J^{* 3}+D^{2} J^{* 2} * D J^{* 2} * J^{* 2} \\
& \left.+D^{2} J * D J^{* 4}+D^{2} J^{* 2} * \mathrm{Rm}+D^{2} J * D J * D \mathrm{Rm}\right)
\end{aligned}
$$




$$
\begin{aligned}
\leq \triangle & \left|D^{2} J\right|+\frac{|D| D^{2} J||^{2}}{\left|D^{2} J\right|}-\frac{\left|D^{3} J\right|^{2}}{\left|D^{2} J\right|} \\
& +C\left(\left|D^{2} J\right|^{2}+\frac{\left|D^{3} J\right|}{t^{1 / 2}}+\frac{\left|D^{2} J\right|}{t}+\frac{1}{t^{2}}+\frac{|D \mathrm{Rm}|}{t^{1 / 2}}\right) .
\end{aligned}
$$

Consider

$$
G(t)=t^{2}\left|D^{2} J\right|+\mu t^{2}|D J|^{2}+t^{3}|\mathrm{Rm}|^{2},
$$

where $\mu$ is a large constant to be determined.

Then, for $\left|D^{2} J\right| \neq 0$,

$$
\begin{aligned}
\frac{\partial}{\partial t} G \leq \triangle & -t^{2} \frac{\left|D^{3} J\right|^{2}}{\left|D^{2} J\right|}-2 \mu t^{2}\left|D^{2} J\right|^{2}-2 t^{3}|D \mathrm{Rm}|^{2} \\
& +C\left(t^{2}\left|D^{2} J\right|^{2}+t^{3 / 2}\left|D^{3} J\right|+\mu t\left|D^{2} J\right|+\mu+t^{3 / 2}|D \mathrm{Rm}|\right) \\
& +\left\langle D\left|t^{2} D^{2} J\right|, \frac{D\left|D^{2} J\right|}{\left|D^{2} J\right|}\right\rangle \\
\leq \triangle & G-\frac{1}{2} t^{2} \frac{\left|D^{3} J\right|^{2}}{\left|D^{2} J\right|}-\frac{1}{2} t^{2}\left|D^{2} J\right|^{2}-\frac{1}{2} t^{3}|D \mathrm{Rm}|^{2} \\
& +\left\langle D\left|t^{2} D^{2} J\right|, \frac{D\left|D^{2} J\right|}{\left|D^{2} J\right|}\right\rangle+C,
\end{aligned}
$$

where $\mu$ is determined now.

Then

$$
\begin{aligned}
\frac{\partial}{\partial t} G \leq \triangle & -\frac{1}{2} t^{2} \frac{\left|D^{3} J\right|^{2}}{\left|D^{2} J\right|}-\frac{1}{2} t^{2}\left|D^{2} J\right|^{2}-\frac{1}{2} t^{3}|D \mathrm{Rm}|^{2}+C \\
+ & \left\langle D G, \frac{D\left|D^{2} J\right|}{\left|D^{2} J\right|}\right\rangle-\mu t^{2}\left\langle D|D J|^{2}, \frac{D\left|D^{2} J\right|}{\left|D^{2} J\right|}\right\rangle-t^{3}\left\langle D|\mathrm{Rm}|^{2}, \frac{D\left|D^{2} J\right|}{\left|D^{2} J\right|}\right\rangle .
\end{aligned}
$$

Notice that

$\left.|D| D J\right|^{2}|\leq| 2\langle D D J, D J\rangle|\leq 2| D^{2} J|| D J|, \quad| D\left|D^{2} J\right|\left|=\frac{\left.|D| D^{2} J\right|^{2} \mid}{2\left|D^{2} J\right|} \leq\right| D^{3} J \mid$.

Hence,

$$
\begin{aligned}
\frac{\partial}{\partial t} G \leq \triangle G-\frac{1}{4} t^{2} \frac{\left|D^{3} J\right|^{2}}{\left|D^{2} J\right|}-\frac{1}{4} t^{2}\left|D^{2} J\right|^{2}-\frac{1}{2} t^{3}|D \mathrm{Rm}|^{2} & +C \\
& +\left\langle D G, \frac{D\left|D^{2} J\right|}{\left|D^{2} J\right|}\right\rangle+C \frac{t^{2}|D \mathrm{Rm}|^{2}}{\left|D^{2} J\right|} .
\end{aligned}
$$

So if we suppose that $\left|D^{2} J\right| \geq 4 C / t$, we have the estimate

$$
\frac{\partial}{\partial t} G \leq \triangle G+\left\langle D G, \frac{D\left|D^{2} J\right|}{\left|D^{2} J\right|}\right\rangle+C,
$$


where $C=C(n, K)$. That is to say, for any $(p, t)$, either we have the estimate $\left|D^{2} J\right| \leq 4 C / t$, or else (18) holds. Let $\bar{G}=G-C t$, where $C$ is chosen suitably. We obtain that either $\bar{G} \leq 0$ or

$$
\frac{\partial}{\partial t} \bar{G} \leq \Delta \bar{G}+\left\langle D \bar{G}, \frac{D\left|D^{2} J\right|}{\left|D^{2} J\right|}\right\rangle .
$$

Notice that $\bar{G}=0$ when $t=0$. Then one may apply the maximum principle to show that $\bar{G} \leq 0$ for every $(p, t)$, which implies the desired estimate. This completes the proof of Theorem 1.2.

Remark 5.4. Theorem 1.2 is scaling-invariant when we replace $g(t)$ by $\bar{g}(t)=$ $c g(t / c)$.

Proof of Theorem 1.3. The argument is standard, as in Ricci flow [Chow and Knopf 2004]. We just sketch the proof.

Suppose not. Then $|\mathrm{Rm}|,|D J|$ are bounded. From Theorem 1.2, all covariant derivatives of Rm and $J$ are bounded. Then we see that the metrics $g$ are uniformly bounded. We fix a coordinate atlas. From the evolution equation of $\Gamma$ and the boundedness of the covariant derivatives of $\mathrm{Rm}$ and $J$, we obtain the boundedness of $\Gamma$. Then we obtain the boundedness of $\partial g, \partial J$, and by induction we see that $\partial^{k} g, \partial^{k} J$ and $\partial^{k} \Gamma$ are bounded. Finally, we obtain that $\left(\partial^{l} / \partial t^{l}\right) \partial^{k} g,\left(\partial^{l} / \partial t^{l}\right) \partial^{k} J$ are bounded. Then, by theorems in mathematical analysis, $(g(t), J(t))$ can be extended to $(g(T), J(T))$ smoothly in all variables of space and time. The almost Hermitian condition is guaranteed by the continuity. Then, from the short-time existence, $(g(t), J(t))$ exists for $t \in[0, T+\epsilon)$, which is a contradiction to the maximality of $T$.

\section{Acknowledgements}

The author wishes to express his gratitude to his advisor Gang Tian, for suggesting to him the problem of constructing new curvature flows preserving generalized complex structure, for encouraging him all the time, and for many helpful discussions. The author would also like to thank Jeffrey Streets for his helpful comments and suggestions, especially for pointing out that this flow may also preserve the integrability of $J$. The author would also like to thank CSC and TRAM for supporting the author visiting Princeton University.

\section{References}

[Boling 2014] J. Boling, "Homogeneous solutions of pluriclosed flow on closed complex surfaces", preprint, 2014. arXiv 1404.7106 
[Chow and Knopf 2004] B. Chow and D. Knopf, The Ricci flow: An introduction, Mathematical Surveys and Monographs 110, Amer. Math. Soc., Providence, RI, 2004. MR 2005e:53101 Zbl 1086.53085

[Enrietti 2013] N. Enrietti, "Static SKT metrics on Lie groups", Manuscripta Math. 140:3-4 (2013), 557-571. MR 3019139 Zbl 1308.32026

[Enrietti et al. 2015] N. Enrietti, A. Fino, and L. Vezzoni, "The pluriclosed flow on nilmanifolds and tamed symplectic forms", J. Geom. Anal. 25:2 (2015), 883-909. MR 3319954 Zbl 06444563

[Fernández-Culma 2013] E. Fernández-Culma, "Soliton almost Kähler structures on 6-dimensional nilmanifolds for the symplectic curvature flow", preprint, 2013. arXiv 1303.5461

[Gauduchon 1997] P. Gauduchon, "Hermitian connections and Dirac operators", Boll. Un. Mat. Ital. $B$ (7) 11:2 (1997), 257-288. MR 98c:53034 Zbl 0876.53015

[Gualtieri 2011] M. Gualtieri, "Generalized complex geometry”, Ann. of Math. (2) 174:1 (2011), 75-123. MR 2012h:53185 Zbl 1235.32020

[Hitchin 2003] N. Hitchin, "Generalized Calabi-Yau manifolds", Q. J. Math. 54:3 (2003), 281-308. MR 2004h:32024 Zbl 1076.32019

[Newlander and Nirenberg 1957] A. Newlander and L. Nirenberg, "Complex analytic coordinates in almost complex manifolds", Ann. of Math. (2) 65 (1957), 391-404. MR 19,577a Zbl 0079.16102

[Perelman 2002] G. Perelman, "The entropy formula for the Ricci flow and its geometric applications", preprint, 2002. arXiv math/0211159

[Pook 2012] J. Pook, "Homogeneous and locally homogeneous solutions to symplectic curvature flow", preprint, 2012. arXiv 1202.1427

[Smith 2013] D. J. Smith, "Stability of the almost Hermitian curvature flow", preprint, 2013. arXiv 1308.6214

[Streets and Tian 2010] J. Streets and G. Tian, "A parabolic flow of pluriclosed metrics", Int. Math. Res. Not. 2010:16 (2010), 3101-3133. MR 2011h:53091 Zbl 1198.53077

[Streets and Tian 2011] J. Streets and G. Tian, "Hermitian curvature flow", J. Eur. Math. Soc. (JEMS) 13:3 (2011), 601-634. MR 2012f:53142 Zbl 1214.53055

[Streets and Tian 2012] J. Streets and G. Tian, "Generalized Kähler geometry and the pluriclosed flow”, Nuclear Phys. B 858:2 (2012), 366-376. MR 2881439 Zbl 1246.53091

[Streets and Tian 2013] J. Streets and G. Tian, "Regularity results for pluriclosed flow", Geom. Topol. 17:4 (2013), 2389-2429. MR 3110582 Zbl 1272.32022

[Streets and Tian 2014] J. Streets and G. Tian, "Symplectic curvature flow", J. Reine Angew. Math. 696 (2014), 143-185. MR 3276165 Zbl 1305.53083

[Vezzoni 2011] L. Vezzoni, "On Hermitian curvature flow on almost complex manifolds", Differential Geom. Appl. 29:5 (2011), 709-722. MR 2012m:53146 Zbl 1225.53030

Received July 4, 2014. Revised September 25, 2014.

\section{SONG DAI}

School of Mathematical Science

PEKING UNIVERSITY

NO. 5 YIHEYUAN ROAD

HAIDIAN DISTRICT

BEIJING, 100871

CHINA

daisong0620@gmail.com 


\title{
PACIFIC JOURNAL OF MATHEMATICS
}

\author{
msp.org/pjm
}

Founded in 1951 by E. F. Beckenbach (1906-1982) and F. Wolf (1904-1989)

\section{EDITORS}

Don Blasius (Managing Editor)

Department of Mathematics

University of California

Los Angeles, CA 90095-1555

blasius@math.ucla.edu

\author{
Paul Balmer \\ Department of Mathematics \\ University of California \\ Los Angeles, CA 90095-1555 \\ balmer@math.ucla.edu \\ Robert Finn \\ Department of Mathematics \\ Stanford University \\ Stanford, CA 94305-2125 \\ finn@math.stanford.edu \\ Sorin Popa \\ Department of Mathematics \\ University of California \\ Los Angeles, CA 90095-1555 \\ popa@math.ucla.edu
}

\author{
Vyjayanthi Chari \\ Department of Mathematics \\ University of California \\ Riverside, CA 92521-0135 \\ chari@math.ucr.edu \\ Kefeng Liu \\ Department of Mathematics \\ University of California \\ Los Angeles, CA 90095-1555 \\ liu@math.ucla.edu \\ Jie Qing \\ Department of Mathematics \\ University of California \\ Santa Cruz, CA 95064 \\ qing@ cats.ucsc.edu
}

\section{PRODUCTION}

Silvio Levy, Scientific Editor, production@msp.org

\section{SUPPORTING INSTITUTIONS}

ACADEMIA SINICA, TAIPEI

CALIFORNIA INST. OF TECHNOLOGY

INST. DE MATEMÁTICA PURA E APLICADA

KEIO UNIVERSITY

MATH. SCIENCES RESEARCH INSTITUTE

NEW MEXICO STATE UNIV.

OREGON STATE UNIV.

\author{
STANFORD UNIVERSITY \\ UNIV. OF BRITISH COLUMBIA \\ UNIV. OF CALIFORNIA, BERKELEY \\ UNIV. OF CALIFORNIA, DAVIS \\ UNIV. OF CALIFORNIA, LOS ANGELES \\ UNIV. OF CALIFORNIA, RIVERSIDE \\ UNIV. OF CALIFORNIA, SAN DIEGO \\ UNIV. OF CALIF., SANTA BARBARA
}

\author{
Daryl Cooper \\ Department of Mathematics \\ University of California \\ Santa Barbara, CA 93106-3080 \\ cooper@math.ucsb.edu \\ Jiang-Hua Lu \\ Department of Mathematics \\ The University of Hong Kong \\ Pokfulam Rd., Hong Kong \\ jhlu@maths.hku.hk \\ Paul Yang \\ Department of Mathematics \\ Princeton University \\ Princeton NJ 08544-1000 \\ yang@math.princeton.edu
}

These supporting institutions contribute to the cost of publication of this Journal, but they are not owners or publishers and have no responsibility for its contents or policies.

See inside back cover or msp.org/pjm for submission instructions.

The subscription price for 2015 is US \$420/year for the electronic version, and \$570/year for print and electronic.

Subscriptions, requests for back issues and changes of subscribers address should be sent to Pacific Journal of Mathematics, P.O. Box 4163, Berkeley, CA 94704-0163, U.S.A. The Pacific Journal of Mathematics is indexed by Mathematical Reviews, Zentralblatt MATH, PASCAL CNRS Index, Referativnyi Zhurnal, Current Mathematical Publications and Web of Knowledge (Science Citation Index).

The Pacific Journal of Mathematics (ISSN 0030-8730) at the University of California, c/o Department of Mathematics, 798 Evans Hall \#3840, Berkeley, CA 94720-3840, is published twelve times a year. Periodical rate postage paid at Berkeley, CA 94704, and additional mailing offices. POSTMASTER: send address changes to Pacific Journal of Mathematics, P.O. Box 4163, Berkeley, CA 94704-0163.

PJM peer review and production are managed by EditFLOW ${ }^{\circledR}$ from Mathematical Sciences Publishers.

\section{PUBLISHED BY}

\section{mathematical sciences publishers \\ nonprofit scientific publishing}

http://msp.org/

(C) 2015 Mathematical Sciences Publishers 


\section{PACIFIC JOURNAL OF MATHEMATICS}

Volume $277 \quad$ No. $2 \quad$ October 2015

The Borel-Weil theorem for reductive Lie groups

JosÉ ARAUJO and TIM BRATTEN

A curvature flow unifying symplectic curvature flow and pluriclosed

287 flow

SONG DAI

Representations of knot groups into $\mathrm{SL}_{n}(\mathbb{C})$ and twisted Alexander polynomials

Michael HeUsener and JoAn PORTi

Approximations by maximal Cohen-Macaulay modules

HENRIK HOLM

Patterson-Sullivan currents, generic stretching factors and the asymmetric Lipschitz metric for outer space

ILYA KAPOVICH and MARTIN LUSTIG

On recurrence over subsets and weak mixing

JiAn Li, Piotr Oprocha and GUOHUA Zhang

Représentations de Steinberg modulo $p$ pour un groupe réductif sur un 425 corps local

TONY LY

Calculating two-strand jellyfish relations

DAVID PENNEYS and EMILY PETERS 